\title{
Charmonium decays at BESIII
}

\author{
Bo Zheng* (for BESIII Collaboration) ${ }^{\dagger}$ \\ University of South China, Hengyang 421001, People's Republic of China \\ Helmholz-Institut Mainz, Johann-Joachim-Becher-Weg 45, D-55099 Mainz, Germany \\ E-mail: zhengbo_uscd163.com
}

The BESIII Experiment at the Beijing Electron Positron Collider (BEPCII) has accumulated the largest $e^{+} e^{-}$collisions data sets in the $\tau$-charm region in the world. Using the data sets of 448.1 million $\psi(3686)$ events and 1.3 billion $J / \psi$ events, the branching fractions and the angular distributions of $J / \psi$ and $\psi(3686)$ decay to $\Lambda \bar{\Lambda}, \Sigma^{0} \bar{\Sigma}^{0}, \Sigma(1385)^{0} \bar{\Sigma}(1385)^{0}$ and $\Xi^{0} \bar{\Xi}^{0}$ are measured. The improved branching fractions $\psi(3686) \rightarrow \gamma \pi^{0}, \gamma \eta, \gamma \eta^{\prime}$ are reported. The Dalitz decays of $\psi(3686) \rightarrow e^{+} e^{-} \chi_{c J}$ and $\chi_{c J} \rightarrow e^{+} e^{-} J / \psi$ are observed and the branching fractions are measured. The Dalitz plot shows the point-like behavior of $\chi_{c J}$. The higher-order multipole amplitudes in $\psi(3686) \rightarrow \gamma \chi_{c 1,2}$ with $\chi_{c 1,2} \rightarrow \gamma J / \psi$ are measured, as a byproduct the $\eta_{c}(2 S) \rightarrow \gamma J / \psi$ transition is searched. The normalized magnetic-quadrupole (M2) amplitude for $\psi(3686) \rightarrow \gamma \chi_{c 1,2}$ with $\chi_{c 1,2} \rightarrow \gamma \gamma J / \psi$ and the normalized electricoctupole (E3) amplitudes for $\psi(3686) \rightarrow \gamma \chi_{c 2}$, $\chi_{c 2} \rightarrow \gamma J / \psi$ are determined. With $\psi(3686)$ radiative decays, many new $\chi_{c J}$ decays are observed for the first time, such as $\chi_{c 0,2} \rightarrow \eta \eta^{\prime}, \chi_{c 2} \rightarrow \eta^{\prime} \eta^{\prime}, K(892)^{*} K$ and $\chi_{c J} \rightarrow \Sigma^{+/ 0} \bar{\Sigma}^{-/ 0}$. Improved measurements of $\eta_{c} \rightarrow \phi \phi$ and searches for $\eta_{c} \rightarrow \omega \phi$ and $\eta_{c} / \eta(1405) \rightarrow \pi^{+} \pi^{-} \pi^{0}$ are reported.

XVII International Conference on Hadron Spectroscopy and Structure - Hadron2017

25-29 September, 2017

University of Salamanca, Salamanca, Spain

\footnotetext{
* Speaker.

$\dagger$ The speaker would like to acknowledge financial supports from the Helmholtz Institute Mainz, the China Scholarship Council, and the National Natural Science Foundation of China (NSFC) under Contract Nos. 11575077 and 11475090 .
} 


\section{Introduction}

The BESIII detector [四] at the BEPCII [四] double-ring $e^{+} e^{-}$collider is designed for the studies of physics in the $\tau$-charm energy region. The peak luminosity of BEPCII is $1.0 \times 10^{33} \mathrm{~cm}^{-2} \mathrm{~s}^{-1}$ at $3773 \mathrm{MeV}$, which has been achieved at April, 2016. The BESIII detector consists of a main drift chamber, an electro-magnetic calorimeter, a time-of-flight system and a muon counter. The details of the BESIII detector can be found in Ref. [四]. The BESIII detector has accumulated 448.1 million $\psi(3686)$ and 1.3 billion $J / \psi$ events. With these world largest $J / \psi$ and $\psi(3686)$ data sets, many charmoinium decays are observed for the first time or measured with the highest precision.

\section{Measurement of $J / \psi, \psi(3686)$ and $\chi_{c J}$ decay to $B \bar{B}$}

The decays of the charmonium resonances $J / \psi$ and $\psi(3686)$ into baryon anti-baryon pairs $(B \bar{B})$ in $e^{+} e^{-}$annihilation have been extensively studied to test the perturbative quantum chromodynamics (QCD) [D], 12\% rule, isospin symmetry and helicity selection rule. The angular distribution of baryons from $e^{+} e^{-} \rightarrow J / \psi(\psi(3686)) \rightarrow B \bar{B}$ is expected to be

$$
\frac{d N}{d \cos \theta}=1+\alpha \cos ^{2} \theta
$$

where $\theta$ is the angle between the baryon and the beam direction in the $e^{+} e^{-}$center-of-mass system. The $\alpha$ is expected to be positive in most of models.

The measurements of $J / \psi$ and $\psi(3686)$ decay to $\Sigma(1385)^{0} \bar{\Sigma}(1385)^{0}$ and $\Xi^{0} \bar{\Xi}^{0}$ are performed [B]] via a single tag technique, in which the baryon $\Sigma(1385)^{0} / \Xi^{0}$ is reconstructed without including the anti-baryon mode due to imperfection of the simulation related to the effect of annihilation for anti-proton. The $\Sigma(1385)^{0} / \Xi^{0}$ is reconstructed by $\Sigma(1385)^{0} / \Xi^{0} \rightarrow \pi^{0} \Lambda$ with the $\Lambda \rightarrow p \pi^{-}$and $\pi^{0} \rightarrow \gamma \gamma$. The $\chi_{c J} \rightarrow \Sigma^{0} \bar{\Sigma}^{0}, \Sigma^{+} \bar{\Sigma}^{-}$are studied [䧃] with full reconstruction. The measured branching fractions and $\alpha$ are summarized in Tabs. 回, 口, and B, respectively.

Table 1: The branching fractions for $J / \psi\left(\psi(3686) \rightarrow B \bar{B}\right.$ (in units of $\left.10^{-4}\right)$. The first uncertainties are statistical, and the second systematic.

\begin{tabular}{lcc}
\hline Mode & $\mathscr{B}(J / \psi \rightarrow)$ & $\mathscr{B}(\psi(3686) \rightarrow)$ \\
\hline$\Lambda \bar{\Lambda}$ & $19.43 \pm 0.03 \pm 0.33$ & $3.97 \pm 0.02 \pm 0.12$ \\
$\Sigma^{0} \bar{\Sigma}^{0}$ & $11.64 \pm 0.04 \pm 0.23$ & $2.44 \pm 0.03 \pm 0.11$ \\
$\Sigma(1385)^{0} \bar{\Sigma}(1385)^{0}$ & $10.71 \pm 0.09 \pm 0.82$ & $0.69 \pm 0.05 \pm 0.05$ \\
$\Xi^{0} \bar{\Xi}^{0}$ & $11.65 \pm 0.04 \pm 0.43$ & $2.73 \pm 0.03 \pm 0.13$ \\
\hline
\end{tabular}

To test $12 \%$ rule, the ratio of $\psi(3686)$ to $J / \psi$ decay to these channels are calculated, which are $(20.43 \pm 0.11 \pm 0.58) \%,(20.96 \pm 0.27 \pm 0.92) \%,(6.44 \pm 0.47 \pm 0.64) \%$, and $(23.43 \pm 0.26 \pm$ $1.09) \%$ for $\Lambda \bar{\Lambda}$ and $\Sigma^{0} \bar{\Sigma}^{0}, \Sigma(1385)^{0} \bar{\Sigma}(1385)^{0}$, and $\Xi^{0} \bar{\Xi}^{0}$, respectively. All the ratios are not in agreement with $12 \%$.

To test isospin symmetry, the ratios of the branching fractions are calculated based on the measurements between the neutral mode and the corresponding charged modes [\$] taking into account the cancelation of the common systematic uncertainties. All ratios are within $1 \sigma$ of the expectation of isospin symmetry. 
Table 2: The branching fractions for $\chi_{c J} \rightarrow B \bar{B}$ (in units of $10^{-5}$ ). The first uncertainties are statistical, and the second systematic.

\begin{tabular}{lccc}
\hline Decay mode & $\mathscr{B}\left(\chi_{c 0} \rightarrow\right)$ & $\mathscr{B}\left(\chi_{c 1} \rightarrow\right)$ & $\mathscr{B}\left(\chi_{c 2} \rightarrow\right)$ \\
\hline$\Sigma^{+} \bar{\Sigma}^{-}$ & $51.8 \pm 2.6 \pm 3.0$ & $3.8 \pm 0.6 \pm 0.3$ & $3.6 \pm 0.7 \pm 0.3$ \\
$\Sigma^{0} \bar{\Sigma}^{0}$ & $47.7 \pm 1.9 \pm 3.6$ & $3.7 \pm 1.0 \pm 0.5$ & $3.8 \pm 1.0 \pm 0.5$ \\
\hline
\end{tabular}

Table 3: The measured $\alpha$ values for $J / \psi(\psi(3686) \rightarrow B \bar{B}$. The first uncertainties are statistical, and the second systematic.

\begin{tabular}{lcc}
\hline Decay mode & $J / \psi \rightarrow$ & $\psi(3686) \rightarrow$ \\
\hline$\Lambda \bar{\Lambda}$ & $0.469 \pm 0.026 \pm 0.008$ & $0.82 \pm 0.08 \pm 0.02$ \\
$\Sigma^{0} \bar{\Sigma}^{0}$ & $-0.449 \pm 0.020 \pm 0.008$ & $0.71 \pm 0.11 \pm 0.04$ \\
$\Sigma(1385)^{0} \bar{\Sigma}(1385)^{0}$ & $-0.64 \pm 0.03 \pm 0.10$ & $0.59 \pm 0.25 \pm 0.25$ \\
$\Xi^{0} \bar{\Xi}^{0}$ & $0.66 \pm 0.03 \pm 0.05$ & $0.65 \pm 0.09 \pm 0.14$ \\
\hline
\end{tabular}

\section{Improved measurements of $\psi(3686) \rightarrow \gamma \eta^{\prime}, \gamma \eta$ and $\gamma \pi^{0}$}

The predicted branching fractions of $J / \psi$ and $\psi(3686)$ radiative decay to light hadrons are about $6 \%$ and $1 \%$ [焑], respectively, while only about $10 \%$ of them have been observed exclusively [ []]. charmonium state radiative decays to a pseudoscalar meson are explained by various phenomenological mechanisms [8]. In addition, the ratio $R_{J / \psi}=\frac{\mathscr{B}(J / \psi \rightarrow \gamma \eta)}{\mathscr{B}\left(J / \psi \rightarrow \gamma \eta^{\prime}\right)}$ is predicted to be approximately equal to the ratio of $R_{\psi(3686)}$, which is similarly defined, in the first-order perturbative QCD calculation $[$ [Q].

The BESIII collaboration has reported the measurements of $\psi(3686) \rightarrow \gamma \eta^{\prime}, \gamma \eta$ and $\gamma \pi^{0}$ with a data set containing 106 million $\psi(3686)$ events [ए]]. Improved measurements of these channels with a total of 448 million $\psi(3686)$ events are performed. To increase statistics and suppress background, the $\eta \rightarrow \pi^{+} \pi^{-} \pi^{0}$ and $\eta \rightarrow \pi^{0} \pi^{0} \pi^{0}$ are selected to reconstruct $\gamma \eta$ channel, while $\eta^{\prime}$ is reconstructed by $\eta^{\prime} \rightarrow \eta \pi^{+} \pi^{-}$and $\eta \pi^{0} \pi^{0}$ with $\eta \rightarrow \gamma \gamma$. The $\pi^{0}$ meson is reconstructed with $\pi^{0} \rightarrow \gamma \gamma$.

Clear signals of $\psi(3686) \rightarrow \gamma \eta^{\prime}, \gamma \eta$ and $\gamma \pi^{0}$ are observed, and the statistical significance for $\psi(3686) \rightarrow \gamma \eta$ and $\gamma \pi^{0}$ are $7.3 \sigma$ and $6.7 \sigma$, respectively. Their branching fractions are measured to be $(125.1 \pm 2.2 \pm 6.2) \times 10^{-6},(0.85 \pm 0.18 \pm 0.04) \times 10^{-6}$ and $(0.95 \pm 0.16 \pm 0.05) \times 10^{-6}$ for $\psi(3686) \rightarrow \gamma \eta^{\prime}, \gamma \eta$ and $\gamma \pi^{0}$, respectively, which are consistent with previous BESIII measurements but in higher precision. The $R_{\psi(3686)}=(0.66 \pm 0.13 \pm 0.02) \%$ is about 30 times smaller than $R_{J / \psi}=(21.4 \pm 0.9) \%$, which is the corresponding ratio from $J / \psi$ radiative decays. Further investigations are necessary to understand this difference and the branching fractions of $J / \psi$ and $\psi(3686)$ radiative decay to pseudoscalar meson globally. These results provide an ideal benchmark for testing various theoretical models of radiative decays of $c \bar{c}$ bound states.

\section{Measurement of higher-order multipole amplitudes in $\psi(3686) \rightarrow \gamma \chi_{c 1,2}$}

The electric-dipole (E1) amplitudes are known to be dominant in $\psi(3686) \rightarrow \gamma \chi_{c 1,2}$ and 
$\chi_{c 1,2} \rightarrow \gamma J / \psi$. However, higher multipole amplitudes, such as magnetic quadrupole (M2) and electric-octupole (E3), are allowed for in these transitions, and can provide information on the anomalous magnetic moment $\kappa$ of the charm quark and on the admixture of $S$ - and $D$-wave states.

By assuming $m_{c}=1.5 \mathrm{GeV} / c^{2}$ and ignoring the mixing of $S$ - and $D$-wave states, the contributions $b_{2}^{1,2}$ and $a_{2}^{1,2}$ corrected to first order in $E_{\gamma_{1,2}} / m_{c}$ are predicted to be $b_{2}^{1}=0.029(1+\kappa)$, $a_{2}^{1}=-0.065(1+\kappa), b_{2}^{2}=0.029(1+\kappa)$, and $a_{2}^{2}=-0.096(1+\kappa)$, respectively [U]].

The ratio $b_{2}^{1} / b_{2}^{2}$ and $a_{2}^{1} / a_{2}^{2}$ are predicted to be $(1.000 \pm 0.015)$ and $(0.676 \pm 0.071)$, respectively. Higher-order multipole amplitudes can be obtained by investigating the angular distributions of the particles in the final states. The CLEO experiment reported significant M2 contributions in $\psi(3686) \rightarrow \gamma \chi_{c 1}$ and $\chi_{c 1,2} \rightarrow \gamma J / \psi$ decays [ㅍ] ] and the BESIII found evidence for the M2 contribution in $\psi(3686) \rightarrow \gamma \chi_{c 2}$ and $\chi_{c 2} \rightarrow \pi^{+} \pi^{-} / K^{+} K^{-}$[ए2].

This analysis make use of a joint distributions of the five helicity angles in the final states to measure the high-order multipole amplitudes. The PDFs for the joint angular distribution of the $\chi_{c 1,2}$ decay sequences are defined as $\frac{W_{c J}\left(\theta_{1}, \theta_{2}, \phi_{2}, \theta_{3}, \phi_{3}, a_{2,3}^{J}, b_{2,3}^{J}\right)}{\overline{W_{c J}\left(a_{2,3}^{J}, b_{2,3}^{J}\right)}}$, where $W_{c J}\left(\theta_{1}, \theta_{2}, \phi_{2}, \theta_{3}, \phi_{3}, a_{2,3}^{J}, b_{2,3}^{J}\right)$ is obtained from the helicity amplitues and the Clebsch-Gordan relation [[3] and $\overline{W_{c J}\left(a_{2,3}^{J}, b_{2,3}^{J}\right)}$ is used for normalization.

Nonpure E1 transition for the decay $\psi(3686) \rightarrow \gamma_{1} \chi_{c 1,2} \rightarrow \gamma_{1} \gamma_{2} J / \psi$ is observed with significance $24.3 \sigma$ and $13.4 \sigma$ for $\chi_{c 1}$ and $\chi_{c 2}$ channels [[4]], respectively. The measured result$\mathrm{s}$ are $a_{2}^{1}=-0.0740 \pm 0.0033 \pm 0.0034$ and $b_{2}^{1}=0.0229 \pm 0.0039 \pm 0.0027$ for $\chi_{c 1}$ and $a_{2}^{2}=$ $-0.120 \pm 0.013 \pm 0.004, b_{2}^{2}=0.017 \pm 0.008 \pm 0.002, a_{3}^{2}=-0.013 \pm 0.009 \pm 0.004$ and $b_{3}^{2}=$ $-0.014 \pm 0.007 \pm 0.004$ for $\chi_{c 2}$, respectively. Figure $\square$ shows the comparison of the results with previous measurements with $m_{c}=1.5 \mathrm{GeV} / c^{2}$ and $\kappa=0$. Our results are consistent but with higher precision than those measured by CLEO-c [W]. The ratios of M2 contributions of $\chi_{c 1}$ to $\chi_{c 2}$, which are independent of $m_{c}$ and $\kappa$ at the leading order, are determined to be $b_{2}^{1} / b_{2}^{2}=1.35 \pm 0.72$ and $a_{2}^{1} / a_{2}^{2}=0.617 \pm 0.083$, which are consistent with the theoretical predications mentioned previously.

Based on the measurements, the product branching fractions for $\psi(3686) \rightarrow \gamma \chi_{c 0,1,2} \rightarrow \gamma \gamma J / \psi$ to be $(15.8 \pm 0.3 \pm 0.6) \times 10^{-4},(351.8 \pm 1.0 \pm 12.0) \times 10^{-4}$, and $(199.6 \pm 0.8 \pm 7.0) \times 10^{-4}$, respectively. The resuls for $\chi_{c 0}$ is consistent with previous BESIII measurement, while $3 \sigma$ larger than the result from CLEO [ $\square]$ and the world average value [ $[\mathbf{]}]$. The results for $\chi_{c 1,2}$ are consistent with other measurements.

The search for $\eta_{c}(2 S) \rightarrow \gamma J / \psi$ is a byproduct and no statistically significant signal is observed, and the upper limit is set to be $\mathscr{B}\left(\psi(3686) \rightarrow \gamma \eta_{c}(2 S)\right) \times \mathscr{B}\left(\eta_{c}(2 S) \rightarrow \gamma J / \psi<9.7 \times 10^{-6}\right.$ at the 90\% C.L..

\section{Observation of $\psi(3686) \rightarrow e^{+} e^{-} \chi_{c J}$ and $\chi_{c J} \rightarrow e^{+} e^{-} J / \psi$}

The electromagnetic (EM) Dalitz decays are widely observed in light-quark meson sector, and plays an important role in revealing the structure of hadrons and the interactions between photons and hadrons. However, the analyogous transitions in charmonium decays haven't been studied previously. The measured small contribution of higher-order multipole amplitudes in $\psi(3686) \rightarrow \gamma \chi_{c J}$ requires other explanations on the puzzling discrepancies in the decay branch- 


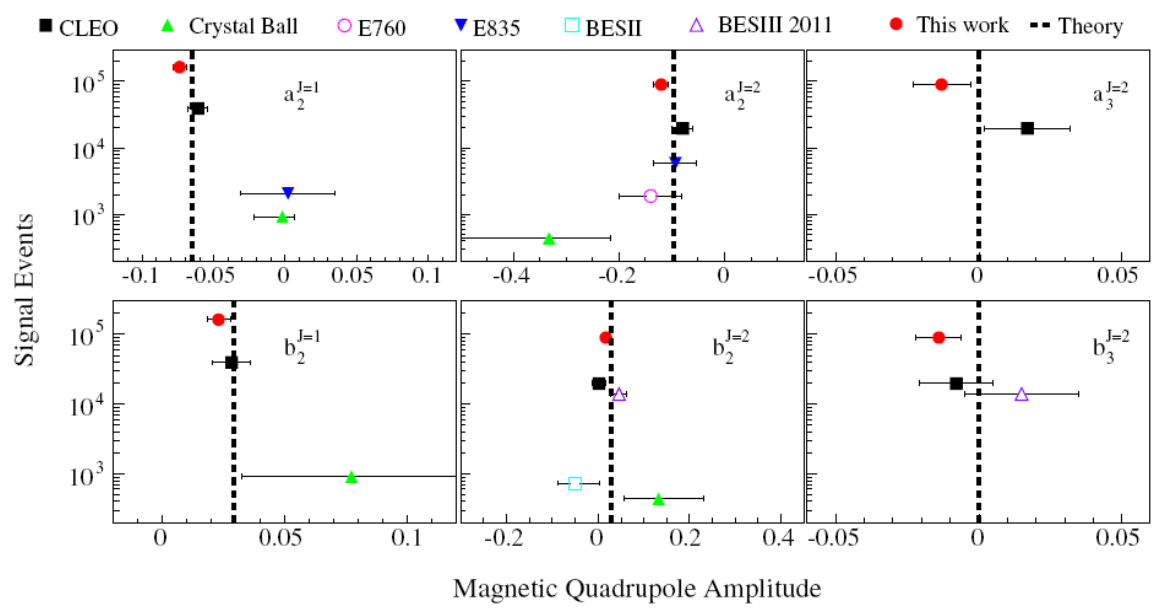

Figure 1: Normalized M2 and E3 amplitudes compared with previous experimental results and theoretical predictions [[ए]] with $m_{c}=1.5 \mathrm{GeV} c^{2}$ and $\kappa=0$. The $y$ axis shows the number of signal events of each experiment.

ing fractions $\mathscr{B}\left(\psi(3686) \rightarrow \gamma \chi_{c J}\right)$ between experimental results and theoretical predictions [ए6]. The $q^{2}$ dependent EM transition form factors (TFFs) of the charmonium states, which can be accessed by the EM Dalitz decays, can provide additional information on the interactions between the charmonium states and the electromagnetic field, serve as a sensitive probe to their internal structures, possibly distinguish the transition mechanisms based on the $c \bar{c}$ scenario and other solutions which alter the simple quark model picture, and probe for exotic hadron structures based on different models.

The measurements of $\psi(3686) \rightarrow e^{+} e^{-} \chi_{c J}$ and $\chi_{c J} \rightarrow e^{+} e^{-} J / \psi$ are performed by analyzing the cascade decays $\psi(3686) \rightarrow e^{+} e^{-} \chi_{c J}, \chi_{c J} \rightarrow \gamma J / \psi$ and $\psi(3686) \rightarrow \gamma \chi_{c J}, \chi_{c J} \rightarrow e^{+} e^{-} J / \psi$, respectively. Figure $\square$ shows the $M(\gamma J / \psi)$ and $M\left(e^{+} e^{-} J / \psi\right)$ spectra, in which clear $\chi_{c J}$ signals are observed and corresponding to the decays $\psi(3686) \rightarrow e^{+} e^{-} \chi_{c J}$ and $\chi_{c J} \rightarrow e^{+} e^{-} J / \psi$. The corresponding branching fractions are measured to be $(11.7 \pm 2.5 \pm 1.0) \times 10^{-4},(8.6 \pm 0.3 \pm 0.6) \times$ $10^{-4}$, and $(6.9 \pm 0.5 \pm 0.6) \times 10^{-4}$ for $\psi(3686) \rightarrow e^{+} e^{-} \chi_{c 0,1,2}$, and $(1.51 \pm 0.30 \pm 0.13) \times 10^{-4}$, $(3.73 \pm 0.09 \pm 0.25) \times 10^{-3}$, and $(2.48 \pm 0.08 \pm 0.16) \times 10^{-4}$ for $\chi_{c 0,1,2} \rightarrow e^{+} e^{-} J / \psi$ [प]], respectively.

The $q$ distributions for the decays $\psi(3686) \rightarrow e^{+} e^{-} \chi_{c 1,2}$ and $\chi_{c 1,2} \rightarrow e^{+} e^{-} J / \psi$ from data are shown in Fig. [1, which are consistent with the distributions from MC samples based on an assumption of pointlike meson model proposed in Ref. [ए8].

\section{Measurement of $\chi_{c 0,2} \rightarrow \eta \eta^{\prime}$ and $\eta^{\prime} \eta^{\prime}$}

Measurements of the the two-body decays of $\chi_{c J}$ to $S S, P P$ and $V V$ ( $S=$ scalar, $P=$ pseudoscalar, $V=$ vector), can reveal the OZI rule violating mechanisms in charmonium sector. It was shown that apart from the singly OZI (SOZI) disconnected process, the doubly OZI (DOZI) disconnected process may play a crucial role in the production of isospin-0 light meson pairs, for instance, in $\chi_{c J} \rightarrow f_{0} f_{0}^{\prime}, \omega \omega, \phi \phi, \omega \phi, \eta \eta, \eta \eta^{\prime}$ and $\eta^{\prime} \eta^{\prime}$. By defining the relative strength $r$ be- 

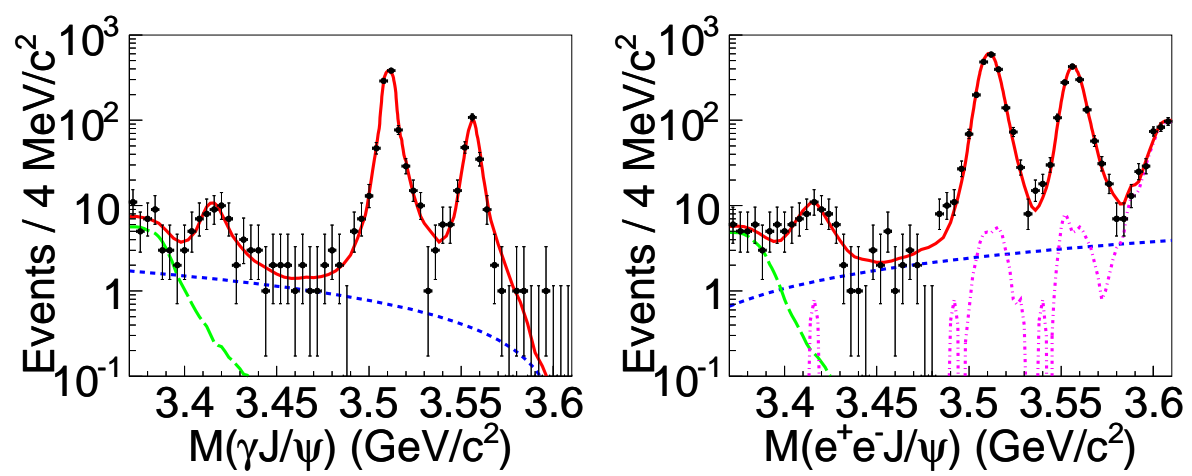

Figure 2: Distributions of (left) $M(\gamma J / \psi)$ and (right) $M\left(e^{+} e^{-} J / \psi\right)$ from data (points with error bars).
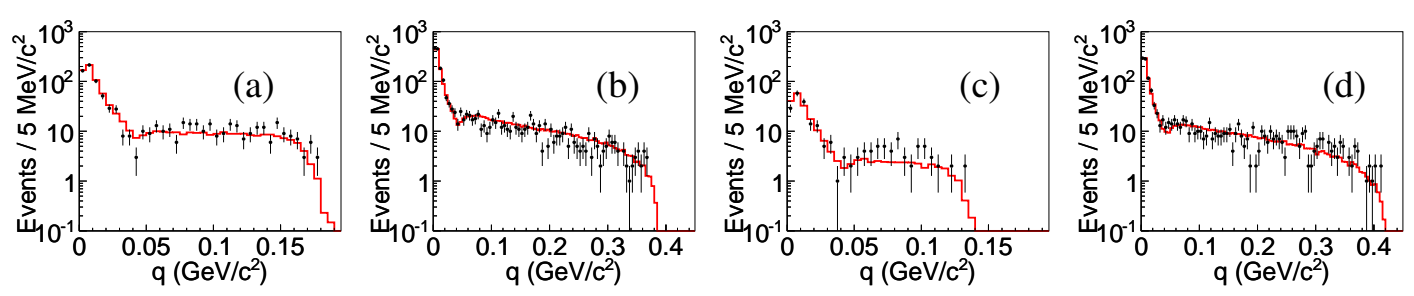

Figure 3: Comparisons of $\mathrm{q}$ distribution between data and MC simulation for the decays (a) $\psi(3686) \rightarrow$ $e^{+} e^{-} \chi_{1}$, (b) $\psi(3686) \rightarrow e^{+} e^{-} \chi_{c 2}$, (c) $\chi_{c 1} \rightarrow e^{+} e^{-} J / \psi$, and (d) $\chi_{c 2} \rightarrow e^{+} e^{-} J / \psi$. The points with error bars are data and the red histograms are for the signal MC simulation.

tween the DOZI and SOZI violating amplitudes in addition to several other physical quantities in the SU(3) flavor basis, insights into the mechanisms for producing light meson pairs in charmonium decays can be gained. For $\chi_{c 0,2} \rightarrow P P$ decays, most of them have been well measured except for the processes with final states containing an $\eta^{\prime}$ meson, to which $r$ is more sensitive. Therefore, measurements of $\chi_{c 0,2} \rightarrow \eta^{\prime} \eta^{\prime}$ and $\eta \eta^{\prime}$ are desirable and crucial to disentangle the roles played by OZI violation in charmonium decay.

To increase statistics, two dominant $\eta^{\prime}$ decay modes, $\eta^{\prime} \rightarrow \gamma \pi^{+} \pi^{-}$and $\eta^{\prime} \rightarrow \eta \pi^{+} \pi^{-}$, are considered, while the $\eta$ is reconstructed in its prominent decay mode $\eta \rightarrow \gamma \gamma$. Consequently, there are three decay modes in the study of $\chi_{c 0,2} \rightarrow \eta^{\prime} \eta^{\prime}$ and two decay modes are considered for $\chi_{c 0,2} \rightarrow$ $\eta \eta^{\prime}$. A simultaneous fit to the spectra of $\eta^{\prime} \eta^{\prime}$ and $\eta \eta^{\prime}$ are performed and the results are obtained, which are $(2.19 \pm 0.03 \pm 0.14) \times 10^{-3},(4.76 \pm 0.56 \pm 0.38) \times 10^{-5},(8.92 \pm 0.84 \pm 0.65) \times 10^{-5}$ and $(2.27 \pm 0.43 \pm 0.25) \times 10^{-5}$ for $\chi_{c 0} \rightarrow \eta^{\prime} \eta^{\prime}, \chi_{c 2} \rightarrow \eta^{\prime} \eta^{\prime}, \chi_{c 0} \rightarrow \eta \eta^{\prime}$ and $\chi_{c 2} \rightarrow \eta \eta^{\prime}$ [ए0] respectively. The decays $\chi_{c 2} \rightarrow \eta^{\prime} \eta^{\prime}, \chi_{c 0} \rightarrow \eta \eta^{\prime}$ and $\chi_{c 2} \rightarrow \eta \eta^{\prime}$ are observed for the first time with significance of $9.6 \sigma, 13.4 \sigma$ and $7.5 \sigma$, respectively, With the measured branching fractions, the parameter $r$ for the $\chi_{c 0}$ and $\chi_{c 2}$ decays to $P P$ final states, is estimated to be around -0.15 according to Eq. (15) in Ref. [DO] with its input parameters. This implies that the contribution from the DOZI violating amplitude is suppressed in $\chi_{c 0,2} \rightarrow P P$ decays in comparison with the SOZI ones [ㅁ] . In addition, we find $\mathscr{B}\left(\chi_{c 0} \rightarrow \eta^{\prime} \eta^{\prime}\right) / \mathscr{B}\left(\chi_{c 2} \rightarrow \eta^{\prime} \eta^{\prime}\right) \approx 45$, which is about one order larger than the ratios for other pseudoscalar meson pairs, ranging from 3 to 6 for $\pi^{+} \pi^{-}, \pi^{0} \pi^{0}$, $K^{+} K^{-}, K_{S}^{0} K_{S}^{0}, \eta \eta[\square]$ and $\eta \eta^{\prime}$. This large ratio is expected by the model proposed in Ref. [LD] 
given a relatively suppressed DOZI-violating contribution. This may initiate further studies about the dynamics of $\chi_{c 0,2} \rightarrow P P$.

\section{Observation of HSR suppressed processes $\chi_{c 2} \rightarrow K(892)^{*} K$ and study of $\chi_{c 2} \rightarrow \rho^{ \pm} \pi$}

The fact that the pQCD plays a dominant role in the charmonium sector is well-known, however, there are many hints that the non-perturbative mechanisms can become important. Exclusive decays of the $P$-wave charmonium state $\chi_{c 2} \rightarrow V P$ are ideal for testing the HSR probing the longrange interactions arising from intermediate $D$-meson loop transitions.

A partial wave analysis of the process $\chi_{c 2} \rightarrow K \bar{K} \pi$ and $\pi^{+} \pi^{-} \pi^{0}$ are performed. The branching fractions for $\chi_{c 2}$ decays to $K^{* \pm} K^{\mp}, K^{* 0} \bar{K}^{0}, K_{2}^{* \pm} K^{\mp}, K_{2}^{* 0} \bar{K}^{0}, K_{3}^{* \pm} K^{\mp}, K_{3}^{* 0} \bar{K}^{0}, a_{2}^{0} \pi^{0}$, and $a_{2}^{ \pm} \pi^{\mp}$ are $(1.5 \pm 0.1 \pm 0.2) \times 10^{-4},(1.3 \pm 0.2 \pm 0.2) \times 10^{-4},(15.5 \pm 0.6 \pm 1.2) \times 10^{-4},(13.0 \pm 1.0 \pm$ $1.5) \times 10^{-4},(5.4 \pm 0.5 \pm 0.7) \times 10^{-4},(5.9 \pm 1.6 \pm 1.5) \times 10^{-4},(13.5 \pm 1.6 \pm 3.2) \times 10^{-4}$ and $(18.4 \pm 3.3 \pm 5.5) \times 10^{-4}[2]$ [] , respectively. The relative large $\chi_{c 2} \rightarrow K^{* \pm} K^{\mp}, K^{* 0} \bar{K}^{0}$ branching fractions indicate the HSR violation occurs via the intermediate mesons loops due to the large $U$ spin symmetry breaking while $\chi_{c 2} \rightarrow \rho^{ \pm} \pi^{\mp}$ due to isospin symmetry breaking. These results are crucial for further study of the HSR violation mechanisms.

\section{Improved measurements of $\eta_{c} \rightarrow \phi \phi, \omega \phi$}

The $\eta_{c}$ decays into vector meson pairs is highly suppressed at leading order in QCD due to the HSR, and it is observed by MARKIII [22] with a product branching fraction with $J / \psi \rightarrow \gamma \eta_{c}$ of $(1.02 \pm 0.25 \pm 0.14) \times 10^{-4}$, and the result is confirmed by BES [23] and BESIII [R4] experiments. However, the measured branching fraction is much larger than the theoretical calculations in different scenario [25].

An amplitude analysis is performed to extract the $\eta_{c} \rightarrow \phi \phi$ yield. The amplitude formulas are constructed with the helicity covariant method [26] and the $\eta_{c}$ resonance is parameterized with the Breit-Wigner function multiplied by a damping factor $f(s)=\frac{1}{M^{2}-s-i M \Gamma} \frac{F\left(E_{\gamma}\right)}{F\left(E_{\gamma}^{0}\right)}$. The branching fraction $\mathscr{B}\left(\eta_{c} \rightarrow \phi \phi\right)$ is determined $\left(2.5 \times 0.3_{-0.7}^{+0.3}\right) \times 10^{-3}$ [27] by using $\mathscr{B}\left(J / \psi \rightarrow \gamma \eta_{c}\right)=1.7 \pm$ $0.4) \%$. A search for $\eta_{c} \rightarrow \omega \phi$ is also performed, no significant signal is observed for this channel and the upper limit is set to be $\mathscr{B}\left(\eta_{c} \rightarrow \omega \phi\right)<2.5 \times 10^{-4}$ at $90 \%$ C.L.

\section{Searches for $\eta_{c} / \eta(1405) \rightarrow \pi^{+} \pi^{-} \pi^{0}$}

The $\eta_{c}$ decays dominant through $c \bar{c}$ annihilation into two gluons, which can be used to obtain the gluon dynamics. Its decay also can be used to test isospin symmetry. Although many of two- or three-body hadornic final states have been measured, the three-pion decay has not been observed before.

The decay $\psi(3686) \rightarrow \gamma \eta_{c}, \eta_{c} \rightarrow \pi^{+} \pi^{-} \pi^{0}$ is used to search for $\eta_{c} \rightarrow \pi^{+} \pi^{-} \pi^{0}$. An unbinned maximum likelihood fit to the invariant mass of $\pi^{+} \pi^{-} \pi^{0}$ in the range of $[2.80,3.15] \mathrm{GeV} / c^{2}$ gives no significant $\eta_{c}$ signal and an upper limit is set to be $\mathscr{B}\left(\eta_{c} \rightarrow \pi^{+} \pi^{-} \pi^{0}\right)<5.5 \times 10^{-4}$ [ㄹ] ]. A similar analysis on $\gamma \pi^{+} \pi^{-} \pi^{0}$ final state give an upper limit of $\mathscr{B}(\psi(3686) \rightarrow \gamma \eta(1405)), \eta(1405) \rightarrow$ $\left.f_{0}(980) \pi^{0}, f_{0}(980) \rightarrow \pi^{+} \pi^{-}\right)<5.2 \times 10^{-7}$. 


\section{Summary}

With the large $J / \psi$ and $\psi(3686)$ data sets collected with BESIII detector, the BESIII collaboration has reported many charmonium decays, which provide important information on charmonium production and decay properties. There are still many analysis ongoing and can be expected to be published in near future. More $J / \psi$ and $\psi(3686)$ events are planed to be collected at the BESIII detector and more results for charmonium decays are promising.

\section{References}

[1] M. Ablikim, et al., (BES Collaboration), Nucl. Instrum. Meth. Phys. Res. A 614, 345 (2010).

[2] S.J. Brodsky, G.P. Lepage, Phys. Rev. D 24, (1981) 2848; J. Bolz, P. Kroll, Eur. Phys. J. C 2 (1998) 545; R.G. Ping, H.C. Chiang, B.S. Zou, Phys. Rev. D 66, (2002) 054020.

[3] M. Ablikim, et al. (BESIII Collaboration), Phys. Rev. D 96, (2017) 052003; Phys. Lett. B 770 (2017) 217.

[4] M. Ablikim, et al. (BESIII Collaboration), arxiv:1710.07922.

[5] M. Ablikim, et al., (BESIII Collaboration), Phys. Rev. D 93, (2016) 072003.

[6] D. L. Scharre et al., Phys. Rev. D 23, 43 (1981).

[7] C. Patrignani et al. (Particle Data Group), Chin. Phys. C 40, 100001 (2016).

[8] H. Fritzsch and J. D. Jackson, Phys. Lett. B 66, 365 (1977); K. T. Chao, Nucl. Phys. B 335, 101 (1990); V. L. Chernyak and A. R. Zhitnitsky, Phys. Rep. 112, 173 (1984); G. W. Intemann, Phys. Rev. D 27, 2755 (1983); Q. Zhao, Phys. Lett. B 697, 52 (2011).

[9] V. L. Chernyak and A.R. Zhitmitsky, Phys. Rep. 112, 173(1984).

[10] M. Ablikim et al. (BESIII Collaboration), Phys. Rev. Lett. 105, (2010) 261801.

[11] M. Artuso et al. (CLEO Collaboration), Phys. Rev. D 80, 112003 (2009).

[12] M. Ablikim et al. (BESIII Collaboration), Phys. Rev. D 84, 092006 (2011).

[13] J. L. Rosner, Phys. Rev. D 78, 114011 (2008).

[14] M. Ablikim et al. (BESIII Collaboration), Phys. Rev. D 95, 072004 (2017).

[15] G. Karl, S. Meshkov, and J. L. Rosner, Phys. Rev. Lett. 45, 215 (1980).

[16] E. Eichten, K. Gottfried, T. Kinoshita, K. D. Lane, and T. M. Yan, Phys. Rev. D 21, 203 (1980); N. Brambilla et al., arXiv:hep-ph/0412158; T. Barnes, S. Godfrey, and E. S. Swanson, Phys. Rev. D 72, 054026 (2005); Z. Cao, M. Cleven, Q. Wang, and Q. Zhao, Eur. Phys. J. C 76, 601 (2016).

[17] M. Ablikim et al. (BESIII Collaboration), Phys. Rev. Lett. 118, (2017) 221802.

[18] A. Faessler, C. Fuchs, and M. I. Krivoruchenko, Phys. Rev. C 61, 035206 (2000).

[19] M. Ablikim, et al. (BESIII Collaboration), arxiv:1707.07042.

[20] Q. Zhao, Phys. Rev. D 72, 074001 (2005); Q. Zhao, Phys. Lett. B 659, 221 (2008).

[21] M. Ablikim, et al. (BESIII Collaboration), arxiv:1612.07398, accepted by Phys. Rev. D (RC).

[22] R. M. Baltrusaitis et al. (MARK-III Collaboration), Phys. Rev. Lett. 52, 2126 (1984). 
[23] J. Z. Bai et al. (BES Collaboration), Phys. Lett. B 578, 16 (2004).

[24] M. Ablikim et al. (BES Collaboration), Phys. Rev. D 72, 072005 (2005).

[25] V. L. Chernyak and A. R. Zhitnitsky, Nucl. Phys. B201, 492 (1982); B214, 547(E) (1983); X.-H. Liu and Q. Zhao, Phys. Rev. D 81, 014017 (2010); B. Gong, Y. Jia, and J. X. Wang, Phys. Lett. B 670, 350 (2009); Y. Jia, Phys. Rev. D 78, 054003 (2008); Y. Jia and G. D. Zhao, Chin. Phys. C 23, 765 (1999); P. Sun, G. Hao, and C.-F. Qiao, Phys. Lett. B 702, 49 (2011); M. Benayoun, V. L. Chernyak, and I. R. Zhitnitsky, Nucl. Phys. B 348, 327 (1991); M. Anselmino, F. Murgia, and F. Caruso, Phys. Rev. D 42, 3218 (1990).

[26] S. U. Chung, Phys. Rev. D 57, 431 (1998); 48, 1225 (1993).

[27] M. Ablikim, et al. (BESIII Collaboration), Phys. Rev. D 95, (2017) 092004.

[28] M. Ablikim, et al. (BESIII Collaboration), arxiv:1707.05178, accepted by Phys. Rev. D. 